Editorial

\title{
Widening the spectrum of extra-neuromuscular involvement in sbma: is there a role for brain pathology?
}

Volume 5 Issue I - 2016

Keywords: SBMA; Extra-neuromuscular involvement; Neuropsychological profile

\section{Editorial}

Spinal and Bulbar Muscular Atrophy (SBMA), also known as Kennedy Disease (KD), is an X-linked, adult onset, slowly progressive, lower motor neuron disease characterized by limb and bulbar muscle atrophy and weakness.

The disease is caused by a CAG repeat expansion in the first exon of the androgen receptor gene (AR), with a number of CAG repeats bigger than 38 retained to be pathogenic. ${ }^{2}$ It usually affects only adult males and the CAG repeat size correlates inversely with disease onset, but not with disease progression or severity. ${ }^{3}$ SBMA belongs to the family of polyglutamine diseases, which also includes Huntington's disease, dentatorubral-pallidoluysian atrophy, and spinocerebellar ataxia (SCA) types 1, 2, 3, 6, 7, and 17. Within the family of polyglutamine diseases, SBMA is unique in its gender-specificity, with full disease manifestation restricted to males. Since the disease is ligand (androgen)-dependent, SBMA manifests primarily in males, which have high levels of circulating androgens in the serum, while females are usually asymptomatic. ${ }^{4}$ Indeed, the polyglutamineexpanded AR is converted to a neurotoxic species upon binding to androgens. ${ }^{5}$

From a clinical point of view, the disease is characterized by slowly progressive lower motor neurons (LMN) degeneration in brainstem and spinal cord, which is associated with multisystem involvement manifesting as androgen insensitivity, heart rhythm abnormalities, metabolic syndrome and sensory neuropathy. ${ }^{6}$ The prevalent clinical features of the disease include wasting of proximal limbs muscles with progressive motor impairment, ${ }^{3}$ associated with mild bulbar involvement. Respiratory failure is rare, but anyway possible. Death usually occurs after about 20 years disease duration.

In the last years, the extense of non-neural phenotype in SBMA has been extensively explored. Accumulation of pathological AR has been found in several visceral organs including the brain. Recent studies have focused on the morpho-functional consequences of polyQ-AR accumulation on the central nervous system. Neuropsychological involvement has been investigated in some small studies with partially contrasting results. Two single case report ${ }^{7,8}$ evidenced the presence of alterations referable to fronto-temporal involvement with impaired visuo-spatial and visuo-constructive abilities, visual shortterm memory deficits, and a personality disorder prompting an altered social conduct.

Another more comprehensive study found impairments in attention, executive functions, and verbal memory in four blood-related SBMA patients. ${ }^{9}$ The first systematic study in this field, involving 20 SBMA patients, ${ }^{10}$ showed a predominantly prefrontal pattern of cognitive

\author{
Querin Giorgia, Sorarù Gianni \\ Department of Neurosciences, University
}

\begin{abstract}
Correspondence: Giorgia Querin, MD, Department of Neurosciences, University of Padova, via Giustiniani 5, 35I28 Padova, Italy, Tel 3904982 I6394, Fax 3.90499E+II, Email giorgia.querin@gmail.com
\end{abstract}

Received: July 07, 2016 | Published: July II, 2016

dysfunction, in terms of subtle deficits in executive functions and an altered short and long-term memory, justified by the frontal lobe's involvement in organizing and processing memory content. Moreover, from a clinical viewpoint, it is not uncommon for patients with SBMA to present with peculiar psychological characteristics such as diffidence, marked emotional sensitivity and concentration problems.

Magnetic resonance and electrophysiological studies ${ }^{11}$ showed subclinical abnormalities in the primary motor cortex and other frontal areas of the brain in SBMA patients. More recently, three magnetic resonance imaging (MRI) studies using voxel-based morphometry (VBM) and diffusion tensor imaging (DTI) reported widespread white matter and subtle gray matter changes in the frontal regions. Kassubek et al. ${ }^{12}$ found extensive white matter atrophy in frontal areas and, to a lesser extent, in some subcortical areas, in the cerebellum and in the brainstem. Further research ${ }^{13}$ found widespread white matter changes in the corticospinal tract and in the limbic system, while another study ${ }^{14}$ described multifocal white matter changes, mainly in the brainstem. A recent positron emission tomography (PET) ${ }^{11}$ study also identified hypometabolism in the frontal regions, even without neuron loss, in ten patients with SBMA. Moreover, a recent study from our group, ${ }^{15}$ pointed out that SBMA patients present with a normal cognitive profile, but with an abnormal theory of mind (ToM). ToM can be defined as a "social cognitive skill that refers broadly to the capacity to understand others' mental states, and to appreciate that these may differ from our own" and is strongly related to empathy. ${ }^{16}$ It can be retained a superior frontal function, with the inferior medial prefrontal cortex, the lateral and orbitomedial prefrontal cortex, and the temporal-parietal junction working as the core neural substrate in ToM operations. ${ }^{17}$ From the literature, it seems now quite clear that there is an association between empathy and androgen levels. ${ }^{18}$ The gender-related differences may also reflect developmental differences in brain structure and function due to the influence of fetal testosterone, to which the male fetuses are more exposed than the female ones. ${ }^{19}$ The SBMA patient's insensitivity to testosterone in all 
tissues, including the brain, could therefore work as a protective factor for affective empathy.

Altogether, increasing evidence shows an important multisystem involvement in SBMA. Recently, several studies, described brain involvement with presence of pathologic nuclear inclusions, associated with diffused white matter alterations at MRI studies and neuropsychological impairment involving mainly pre-frontal patterns. Analysis of higher frontal-related functions through ToM tasks confirmed the presence, even if at a subclinical level, of frontal involvement. Interestingly, such abnormalities could be related not only to a diffuse accumulation of the mutated AR, but also with androgen insensitivity influencing empathy and social behavior. Such theory needs to be confirmed through wider studies and will be of extreme importance to define the entity of neuropsychological involvement in SBMA patients and to establish definitely which functions are involved and the entity of such alterations. In the perspective of the every-day clinical practice, such neuropsychological impairment could be related with a reduced quality of life and compliance to follow up and therapies, which could influence clinical outcomes and survival of the patients.

\section{Disclosure}

The authors report no conflict of interest.

\section{Acknowledgements}

Work supported by Association Francaise contre les Myopathies (18722 to G.S.); Telethon-Italy (GUP15009 to G.S.); Italian Health Ministry (RF-2011-02350097).

\section{Conflicts of interst}

None.

\section{References}

1. Kennedy WR, Alter M, Sung JH Progressive proximal spinal and bulbar muscular atrophy of late onset. A sex-linked recessive trait. Neurology. 1968;18(7):671-680.

2. Atsuta N, Watanabe H, Ito $\mathrm{M}$ et al. Natural history of spinal and bulbar muscular atrophy (SBMA): A study of 223 Japanese patients. Brain. 2006;129(6):1446-1455.

3. Fratta P, Nirmalananthan N, Masset L et al. Correlation of clinical and molecular features in spinal bulbar muscular atrophy. Neurology. 2014;82(23):2077-2084.

4. Takeyama K, Ito S, Yamamoto A et al. Androgen-dependent neurodegeneration by polyglutamine-expanded human androgen receptor in Drosophila. Neuron. 2002;35(5): 855-364.

5. Adachi H, Katsuno M, Minamiyama M, et al. Widespread nuclear and cytoplasmic accumulation of mutant androgen receptor in SBMA patients. Brain. 2005;128(3):659-670.
6. Querin G, Bertolin C, Da Re E, et al. Non-neural phenotype of spinal and bulbar muscular atrophy: results from a large cohort of Italian patients. J Neurol Neurosurg Psychiatry. 2015; doi:10.1136/jnnp-2015-311305.

7. Kessler H, Prudlo J, Kraft S, et al. Dementia of frontal lobe type in Kennedy's disease. Amyotroph Lateral Scler Other Motor Neuron Disord. 2005;6(4):250-253.

8. Mirowska-Guzel D, Seniow J, Sułek A, et al. Are cognitive and behavioural deficits a part of the clinical picture in Kennedy's disease? A case study. Neurocase. 2009;15(4):332-337.

9. Guidetti D, Vescovini E, Motti L, et al. X-linked bulbar and spinal muscular atrophy, or Kennedy disease: clinical, neurophysiological, neuropathological, neuropsychological and molecular study of a large family. J Neurol Sci. 1996;135(2):140-148.

10. Soukup G.R, Sperfeld A.D, Uttner I, et al. Frontotemporal cognitive function in $\mathrm{X}$-linked spinal and bulbar muscular atrophy (SBMA): A controlled neuropsychological study of 20 patients. J Neurol. 2009;256(11):1869-1875.

11. Lai TH, Liu RS, Yang BH, et al. Cerebral involvement in spinal and bulbar muscular atrophy (Kennedy's disease): a pilot study of PET. Journal Neurol Sci. 2013;335(1-2):139-144.

12. Kassubek J, Juengling FD, Sperfeld AD. Widespread white matter changes in Kennedy disease: a voxel based morphometry study. J Neurol Neurosurg Psychiatry. 2009;78(11):1209-1212.

13. Unrath A, Müller HP, Riecker A, et al. Whole brain-based analysis of regional white matter tract alterations in rare motor neuron diseases by diffusion tensor imaging. Hum Brain Mapp. 2010;31(11):1727-1740.

14. Pieper CC, Konrad C, Sommer J, et al. Structural changes of central white matter tracts in Kennedy's disease - a diffusion tensor imaging and voxel-based morphometry study. Acta Neurol Scand. 2013;127(5):323-328

15. Di Rosa E, Sorarù G, Kleinbub JR, et al. Theory of mind, empathy and neuropsychological functioning in X-linked Spinal and Bulbar Muscular Atrophy: a controlled study of 20 patients. J Neurol. 2015;262(2):394-401.

16. Torralva T, Kipps CM, Hodges JR, et al. The relationship between affective decision-making and theory of mind in the frontal variant of fronto-temporal dementia. Neuropsychologia. 2007;45(2):342-349.

17. Premack D, Woodruff G. Does the chimpanzee have a theory of mind? Behav Brain Sci. 1968;1(4):515-526.

18. Van Honk J, Schutter DJ, Bos PA, et al. Testosterone administration impairs cognitive empathy in women depending on second-to-fourth digit ratio. Proc Natl Acad Sci U S A. 2011;108(8):3448-3452.

19. Chapman E, Baron-Cohen S, Auyeung B, et al. Fetal testosterone and empathy: evidence from the empathy quotient (EQ) and the "reading the mind in the eyes" test. Soc Neurosci. 2006;1(2):135-148. 